\title{
Comparison of Geometrical Uncertainties in Breast Radiation Therapy with Different Immobilization Methods
}

\author{
Kanan Jassal ${ }^{1,2 *}$, Bisht $\mathbf{S}^{1}$, Kataria $\mathrm{T}^{1}$, Sachdev $\mathrm{K}^{2}$, Choughle $\mathrm{A}^{2}$ and Supe $\mathrm{S}^{3}$
}

${ }^{1}$ Division of Radiation Oncology, Medanta, The Medicity, Gurgaon 122001, India

${ }^{2}$ Department of Physics, Malviya National Institute of Technology, Jaipur 302001, India

${ }^{3}$ Department of Radiation Physics, Kidwai Memorial Institute of Oncology, Bangalore 560029, India

\begin{abstract}
To compare the set-up uncertainties in breast radiation therapy using two different immobilization methods: vacuum cushion (VC) and standard breast board (BB). The data set comprises of 26 breast cases were divided into two groups based on the type of immobilization used for their radiotherapy treatment, either intensity-modulated radiotherapy (IMRT) or volumetric arc modulated radiotherapy (VMAT). The vacuum cushion group consisted of 14 patients with 143 exposures and the breast board group consisted of 12 patients with 120 exposures. Set-up errors in mediolateral (ML), craniocaudal (CC) and anterioposterior (AP) directions were determined using grey scale matching between the baseline reference and online acquired images by cone beam computed tomography $(\mathrm{CBCT})$ scan. Systematic error $(\Sigma)$, random error $(\sigma)$ and mean displacement vector $(M)$ were calculated for both the groups. For Vacuum Cushion group, maximum error observed was $2.75 \mathrm{~mm}$, mean displacement vector was 1.52 $\mathrm{mm}$ and mean value of set-up error in ML, CC and AP direction was $-0.30 \mathrm{~mm},-0.40 \mathrm{~mm}$ and $1.60 \mathrm{~mm}$ respectively. For Breast Board group, maximum error recorded was $3.87 \mathrm{~mm}$, mean displacement vector was $1.72 \mathrm{~mm}$ and mean value of set-up error in $\mathrm{ML}, \mathrm{CC}$ and $\mathrm{AP}$ direction was $0.80 \mathrm{~mm}, 1.70 \mathrm{~mm}$ and $1.50 \mathrm{~mm}$ respectively. Setup errors evaluated from the two immobilization methods did not differ significantly $(p>0.05)$. Thus, introduction of the vacuum cushion (VC) into routine breast radiation therapy was seen to be as efficient as BB setup.
\end{abstract}

Keywords: Set-up errors; Random error; Systematic error; Breast board; Vacuum cushion

\section{Introduction}

In radiation therapy of breast, there is an increased usage of IMRT and VMAT techniques which has improved dose homogeneity for locoregional control of the disease and also reduced radiation toxicity to heart and lung [1,2]. Other cosmetic benefits of such technologies include reduction in radiation induced edema, dermatitis and hyperpigmentation [3-5]. One of the basic requirements for effective delivery of fractionated radiotherapy is correct delivery of each fraction, this results in the target volume receiving the intended dose. Geometric miss could be a major disadvantage with highly conformal, advanced and fractionated radiotherapy techniques. Thus, errors in patient positioning (set up errors) should be dealt with adequately. In such a situation, the immobilization strategy with on-line image guidance and correction during radiation delivery plays an essential role $[3,4,6$ 9]. Accurate localization would allow for maximal sparing of critical structures, reduction of treatment margins and potential for disease control [10-12]. Thus, maximum benefits of IMRT can be obtained if the patient set-up errors are minimized.

Breast is subjected to both translational and rotational set up errors in addition to uncertainties compounded by breathing motion and breast contour shifts due to its pendulous nature. Setup uncertainties emphasize the need of carefully fabricated accurate immobilization system $[13,14]$ and on-line image guidance, where available, can be of immense value. Whole breast and post mastectomy chestwall has been conventionally immobilized on breast board. Vacuum cushion for breast treatment is also a valid alternative.

In our institute, we have been using both, standard breastboard BB (MedTec, Orange city, IA) and vacuum cushion VC (VacLok, Civico Medical solutions, Kalona, IA) as immobilization devices for our breast patients. This study focuses on comparing the translational setup uncertainties in two different immobilization methods that were corrected using on-line image guidance.

\section{Method}

\section{Patient selection, immobilization and simulation}

Twenty six breast cancer patients $(\mathrm{N}=26)$ were selected for this study. Only those patients were randomly selected who were immobilized in breast board/vacloc, had adequate mobility of arms to keep above head during the entire course of radiation therapy. Selected patients underwent radical radiotherapy of breast/chestwall and were treated at Medanta Cancer Institute between January to August 2011. They were immobilized either with standard technique using breast board BB or vacuum cushion VC. Twelve patients were included in breastboard (BB) group $\left(n_{1}=12\right)$ and remaining fourteen were included in vacuum cushion (VC) group $\left(n_{2}=14\right)$. Out of 26 patients, 13 patients were post mastectomy and 13 patients had breast conservative surgery (BCS). The breastboard group consisted of 6 post mastectomy and 6 were post BCS. Similarly, vacuum cushion group consisted of 7 post mastectomy and 7 post BCS group. The study population included patients who were treated for supraclavicular, internal mammary lymph chain and axiliary nodes. Out of twenty-six, 14 patients were left-sided and remaining 12 patients were right sided.

${ }^{*}$ Corresponding author: Kanan Jassal, Division of Radiation Oncology, Medanta, The Medicity, Gurgaon 122001, India, E-mail: kananjassal@gmail.com

Received August 08, 2012; Accepted March 25, 2013; Published March 30, 2013

Citation: Jassal K, Bisht S, Kataria T, Sachdev K, Choughle A, et al. (2013) Comparison of Geometrical Uncertainties in Breast Radiation Therapy with Different Immobilization Methods. J Nucl Med Radiat Ther 4: 140. doi:10.4172/21559619.1000140

Copyright: () 2013 Jassal K, et al. This is an open-access article distributed under the terms of the Creative Commons Attribution License, which permits unrestricted use, distribution, and reproduction in any medium, provided the original author and source are credited. 
The breast volumes for the selected patients ranged between $506.12 \mathrm{cc}$ and $1804.18 \mathrm{cc}$ with the average value as $986.73 \mathrm{cc}$.

All the patients were first reviewed in the out patient department, decision for radical radiotherapy was taken and then patients were taken to mould room. Assignment of the particular type of immobilization device was based on clinical grounds depending on patient comfort with breast board, movement of shoulder post surgery and other clinical factors. Patients were either immobilized on standard breast board or vacuum cushion based on their individual clinical conditions.

Breast board is conventionally used in radiotherapy departments for immobilizing patients with carcinoma of breast. Breast board has components like support for arms, elbow and wrist; hip stop; adjustable neck support and also variable board angles, is shown in figure 1 .

(i) The board is made up of low density foam and carbon fibre. These components ensure the minimum treatment beam attenuation. Even the lateral areas of breast board are designed in such a way that no attenuation takes place within the path of lateral tangential treatment beams. It has excellent indexing system that helps the technologists for relocating the positions of arms, elbows, hands and wrist. BB is simple to use with its ability for reproducing each patient's individual position. Thus, equipment offers a reliable and highly reproducible patient positioning system. Knee cushions were used often with BB for providing comfort to patients as well as helps in preventing longitudinal slip of the patient during treatment.

Vacuum cushion is also commonly used in radiotherapy departments and is shown in figure1.

(ii). These bags are made up of rubber coated Macintosh, and are filled with small sized thermocol/Styrofoam balls. These components provide air equivalence for the incidental treatment beams. The bags are sufficient in size to cover lateral sides of body. Air is driven out of the bag to create vacuum inside the bag while the patient rests on it. Vacuum hold the position of the Styrofoam balls as displaced by the occupancy of patient's body, which are adjusted according to the body contour of the patient. So, the vacuum bags provide a convenient method of creating an individual body shape of the patient in their comfortable treatment position.

(i)Breast board

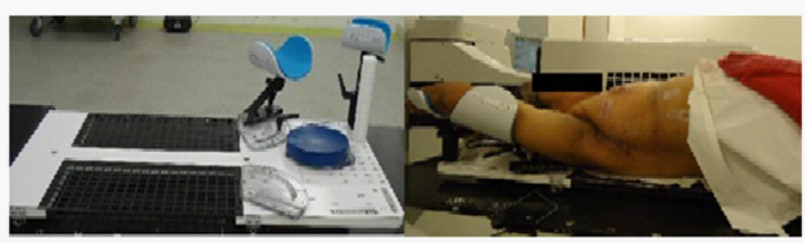

(ii) Vacuum cushion

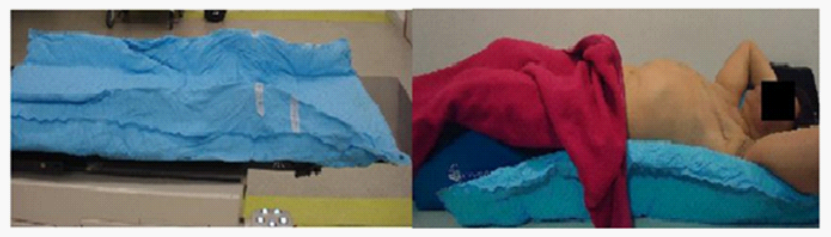

Figure 1: Immobilization devices and respective patient set up.
Patients were positioned with their both arms abducted above the head in either of the immobilization devices. Knee support was also provided to the patients, as per their requirement. Breast conserved patients had their scar identified/ localized on the breast using a radio-opaque marking strip/wire. A one centimeter thick bolus was placed on the scar/surgical bed to build up the dose at skin for post mastectomy patients. Radiation planning CT scan was performed on CT Simulator Siemens SOMATOM" Emotion 16 slice, with contiguous $3 \mathrm{~mm}$ thick slices, starting from base of mandible to L2 vertebrae after placing fiducials according to the departmental protocol (mid axillary line, mid sternum, $2 \mathrm{~cm}$ below breast crease and second intercostals space or upper border of palpable breast). Contouring was done on FocalSim version 4.62. 01 (Elekta CMS, Maryland Heights, USA). Target volume was contoured according to RTOG contouring atlas. A planning target volume (PTV) was generated by giving $5 \mathrm{~mm}$ margins around the clinical target volumes (CTVs). Ipsi-lateral and contralateral lung, contra-lateral breast, heart, spinal cord and humeral heads were contoured as organs at risk. The PTVs were clipped from the skin and were kept $3 \mathrm{~mm}$ below the skin to provide the dose build up region. During the planning, auto flash margins of $1.5 \mathrm{cms}$ were given to breast, in order to account the respiratory movements during delivery.

\section{Radiotherapy planning objectives, techniques and evaluation}

The prescription dose was $5000 \mathrm{cGy}$ in 25 fractions. The objective of planning was to cover $95 \%$ of PTV with $95 \%$ of the prescribed dose and to minimize the volume within PTV receiving dose greater than $107 \%$ and lesser than $93 \%$ of the prescription dose. Dose constraints for OARs were as follows: Heart V25Gy $<5 \%$, mean dose < 10Gy; Ipsilateral lung V20Gy $<20 \%$, mean dose $<12 \mathrm{~Gy}$; contralateral breast mean dose $<3 \mathrm{~Gy}$.

For all the selected patients, radiotherapy planning was done on the Monaco treatment planning system, version 2.03. 01. Static IMRT plans were generated with not more than five static fields of $6 \mathrm{MV}$ photons, while the VMAT plans were designed with a partial arc. The maximum arc swept by the gantry was about 250 degrees in clockwise direction. The constraints were defined using the concept of equivalent uniform dose in Monaco version 2.03. 01, to match the planning objectives. After the fluence generation and segment weight optimization, final dose calculations were done using Monte-carlo algorithm with a spatial resolution of $3 \mathrm{~mm}$. Finally, the treatment plans were evaluated by the radiation oncologist. The target was evaluated for coverage, dose conformity, and dose homogeneity. Dose volume histograms (DVHs) were used for evaluation and comparison of mean dose, V25Gy, V30Gy for heart and similarly mean dose, V5Gy, V10Gy, V20Gy for ipsilateral lung and contralateral breast were also evaluated. Once the plan was found satisfactory on above criterion, it was approved for implementation.

\section{Plan implementation and method of image guidance}

Approved plans were then implemented on the patients as follows. These patients were treated on either of the two linear accelerators (Elekta Synergy-S and Elekta Infinity, Crawley UK) installed at the division of Radiation Oncology at Medanta Cancer Institute, Gurgaon. Both the accelerators have cone beam CT, an X-ray tube and amorphous silicon (a-Si) flat panel detector mounted orthogonally to the accelerator [15]. First day setup of the patient was done as per the planning CT scan. The treatment center and machine isocenter localization shift was done from the CT fiducial markers, the impression of which was preserved on the patient's body. Subsequently, the X-ray volumetric imaging (XVI) was carried out for all the patients before the radiation delivery and once 
in every week during treatment. Cone beam computed tomography (CBCT) scans were acquired within 2 minutes with the $360^{\circ}$ gantry rotation (starting from $+180^{\circ}$ up to $-180^{\circ}$ ) around the patient with the frame rate of $5.5 \mathrm{~Hz}$ and the maximum grabbed frames of 650 [16]. The axial CT images were reconstructed using the projection of the total grabbed frames. The set of CBCT acquired from XVI was matched with the reference $\mathrm{CT}$ on automatic grey scale matching option available in the algorithm of the XVI software as shown in figure 2. Visual inspections of the matched images were also performed in all the axial, coronal and sagittal planes, for ensuring the patient alignment and PTV coverage. The displacements of the patients were recorded. This software reflects the shifts, both translational and roatational. Only the translational shifts were considered in mediolateral (ML), craniocaudal (CC) and anterioposterior (AP) directions and accordingly treatment couch was re-adjusted prior to the treatment. Rotational setup errors were not considered in the present study because the immobilization devices were fixed to the treatment couch and maximum tolerance for such errors in any direction is $\pm 3^{\circ}$. As per our departmental protocol, if the rotational tolerance exceeds, then the patients are repositioned and re-imaged. Within the collected data for all the selected patients, it was observed that rotational differences were lesser than $\pm 1.8^{\circ}$.

Sign conventions used for the movement of couch are shown in table 1. This online image guidance technique is utilized for the real time set-up corrections before the delivery of treatment by both static and dynamic VMAT delivery of the intensity modulated beams. This technique has increased the accuracy and precision in radiotherapy by improving the reproducibility of the patient setup with real time corrections of the errors in setup. The clip-box for all the patients was standardized before the setup by bony anatomy around the PTV as shown in figure 2. A total of 263 acquisitions CBCT were analyzed form 26 numbers of patients. Analysis was performed over 3 months by a single observer.

\section{Statistical analysis}

Simple statistical analysis was done for calculating mean and standard deviations for each of the patients in both the groups. The mean set-up error (m) was computed for each of group. Set up errors

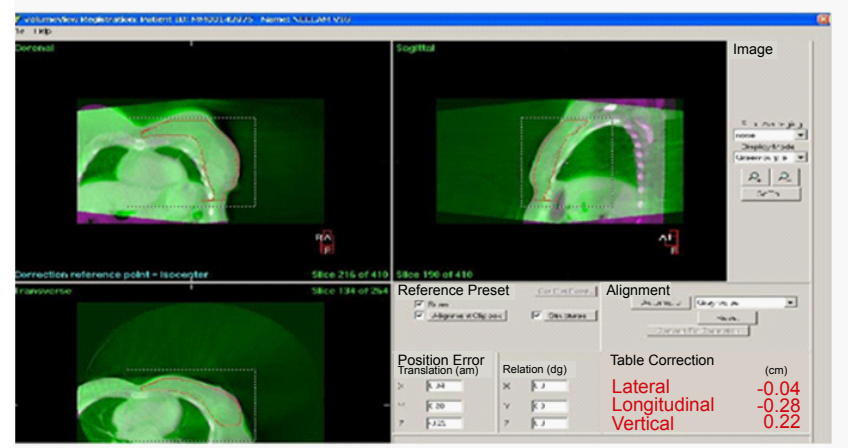

Figure 2: Matching the volumetric cone beam CT with reference CT and clipbox shown.

\begin{tabular}{|c|c|c|c|}
\hline \multirow{2}{*}{ Signs } & \multicolumn{3}{|c|}{ Translational directions for the movement of treatment couch } \\
\cline { 2 - 4 } & Mediolateral (ML) & Craniocaudal (CC) & Anterioposterior (AP) \\
\hline Positive sign & Left & Superior & Up \\
\hline Negative sign & Right & Inferior & Down \\
\hline
\end{tabular}

Table 1: Sign conventions that determine the movement of couch. were divided into two categories: systematic and random errors. Systematic errors are persistent displacements present throughout the entire course of fractionated therapy while random errors vary on a day-to-day basis [13]. Systematic errors $(\Sigma)$ were calculated as the standard deviation of mean errors, calculated for each individual patient in the group. And similarly random errors $(\sigma)$ were computed as the root mean square values of the errors recorded for each patient in each of the groups. The mean displacement vector $(M)$ was also calculated for each cohort.

$$
M=\sqrt{d_{M L}^{2}+d_{C C}^{2}+d_{A P}^{2}}
$$

Where, $\mathrm{d}_{\mathrm{ML}}, \mathrm{d}_{\mathrm{CC}}$ and $\mathrm{d}_{\mathrm{AP}}$ are the deviations in medialateral, craniocaudal and anterioposterior directions.

Statistically, the sample did not satisfy the conditions for normality. Thus, non parametric tests were conducted to find the significant difference between the two groups to compare the effect of different immobilization devices on patients set up. The analysis of data was done in SPSS (SPSS v. 16, IBM, USA) software.

\section{Results}

A total of 263 XVI / CBCT acquisitions were analyzed in 26 patients. Table 2 shows the details of the couch corrections in terms of maximum and mean set-up values in all 3 directions and computed mean displacement vector for each of the cohorts and for complete sample which is termed as population. The group mean $(\mathrm{m})$, systematic error $(\Sigma)$ and random error $(\sigma)$ for BB were: in ML direction $(\mathrm{m}=0.8$ $\mathrm{mm}, \Sigma=2.7 \mathrm{~mm}, \sigma=3.8 \mathrm{~mm})$, CC direction $(\mathrm{m}=1.7 \mathrm{~mm}, \Sigma=2.3 \mathrm{~mm}$, $\sigma=4.3 \mathrm{~mm})$ and in AP direction $(\mathrm{m}=1.5 \mathrm{~mm}, \Sigma=2.1 \mathrm{~mm}, \sigma=3.5$ $\mathrm{mm})$. Similarly, the group mean $(\mathrm{m})$, systematic error $(\Sigma)$ and random error $(\sigma)$ for VC were: in ML direction $(\mathrm{m}=-0.30 \mathrm{~mm}, \Sigma=2.1 \mathrm{~mm}, \sigma$ $=3.3 \mathrm{~mm})$, CC direction $(\mathrm{m}=-0.40 \mathrm{~mm}, \Sigma=2.6 \mathrm{~mm}, \sigma=4.1 \mathrm{~mm})$ and in AP direction $(\mathrm{m}=1.60 \mathrm{~mm}, \Sigma=2.5 \mathrm{~mm}, \sigma=4.5 \mathrm{~mm}$ ) (as tabulated in Tables 2 and 3). The frequency histograms for the BB and VC groups are represented by figures 3 and 4 respectively. The BB group had a larger displacement in CC direction. $12 \%$ of fractions delivered in $\mathrm{BB}$ group and $8 \%$ in $\mathrm{VC}$ group had more than one dimensions outside the maximum threshold that kept at $3 \mathrm{~mm} .15 \%$ of patients in $\mathrm{BB}$ group and $14 \%$ of patients in VC group were observed to have set up displacements greater than $5 \mathrm{~mm}$ in any direction.

With XVI and real time corrections, a significant decrease in systematic and random uncertainties in all mentioned translational directions were observed for both type of immobilization groups. Results from the Mann Whitney \& Wilcoxon Signed Rank Test showed that random errors for the two techniques did not differ significantly $(\mathrm{p}>0.05)$.

\section{Discussions}

Studies conducted for the measurement of amplitude of breast motion during normal breathing show that it varies from patient to patient. For quiet breathing, the range of breast motion has been reported as 5-7 $\mathrm{mm}$ by Kubo et al. [17]. Hence, for advanced radiotherapy techniques particularly for breast treatment, immobilization methods are required to align the patient correctly during treatment delivery [18]. The present study is intended to compare the set up errors using breastboard $\mathrm{BB}$ and vacuum cushion $\mathrm{VC}$ in the routine breast radiotherapy at our facility. Among all the parameters measured, VC demonstrated equivalence to the standard BB. Hence, as far as day-today variability in set up is considered, $\mathrm{VC}$ was observed to be similar to BB settings. 
Citation: Jassal K, Bisht S, Kataria T, Sachdev K, Choughle A, et al. (2013) Comparison of Geometrical Uncertainties in Breast Radiation Therapy with Different Immobilization Methods. J Nucl Med Radiat Ther 4: 140. doi:10.4172/2155-9619.1000140

Page 4 of 6

\begin{tabular}{|c|c|c|c|c|c|}
\hline \multirow{2}{*}{ Category } & $\begin{array}{c}\text { Maximum Error recorded in any irection } \\
\text { (in mm) }\end{array}$ & \multicolumn{3}{|c|}{ Mean Set-up Error (in mm) } \\
\cline { 2 - 5 } & Mediolateral (ML) & Craniocaudal (CC) & Anterioposterior (AP) & Mean Displacement Vector (M) (in \\
Population & 3.87 & 2.1 & 1.5 & 1.16 \\
\hline VC & 2.75 & -0.3 & -0.4 & 1.6 \\
\hline BB & 3.87 & 0.8 & 1.7 & 1.57 \\
\hline
\end{tabular}

Table 2: Maximum, mean set up errors in ML, CC and AP directions and value of mean displacement vector for the population, VC and BB groups.

\begin{tabular}{|l|c|c|c|c|c|c|}
\hline \multirow{2}{*}{ Category } & \multicolumn{3}{|c|}{ Systematic Error $(\Sigma)$, in $\mathbf{~ m m}$} & \multicolumn{2}{c|}{ Random Error $(\sigma)$, in $\mathbf{m m}$} \\
\cline { 2 - 6 } & Mediolateral $(\mathbf{M L})$ & Craniocaudal $(\mathbf{C C})$ & Anterioposterior $(\mathbf{A P})$ & Mediolateral (ML) & $\mathbf{C r a n i o c a u d a l}(\mathbf{C C})$ & Anterioposterior $(\mathbf{A P})$ \\
\hline Population & 3.5 & 3.8 & 2.8 & 2.3 & 2.5 \\
\hline BB & 2.7 & 2.3 & 2.1 & 3.8 & 4.3 \\
\hline VC & 2.1 & 2.6 & 2.5 & 3.3 & 3.1 \\
\hline
\end{tabular}

Table 3: Systematic $(\Sigma)$ and random $(\sigma)$ errors calculated for population, VC and BB groups.
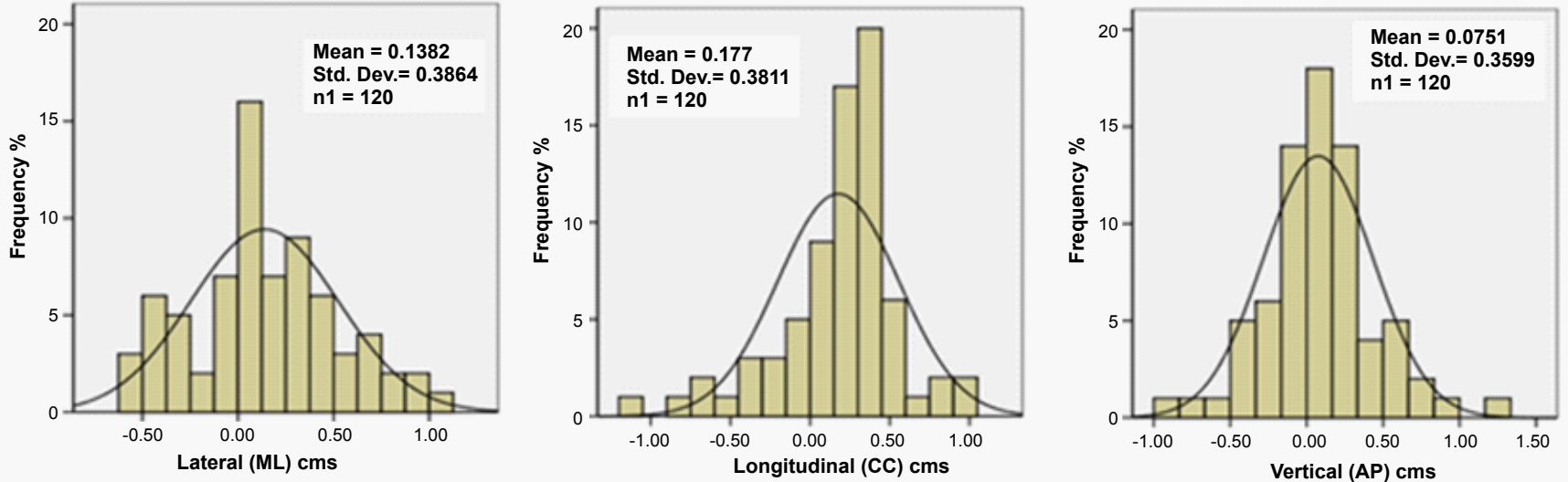

Figure 3: Measured set-up errors on breast board $B B$ in lateral( $M L)$, longitudinal $(C C)$ and vertical( $A P)$ directions with their mean and standard deviation values.
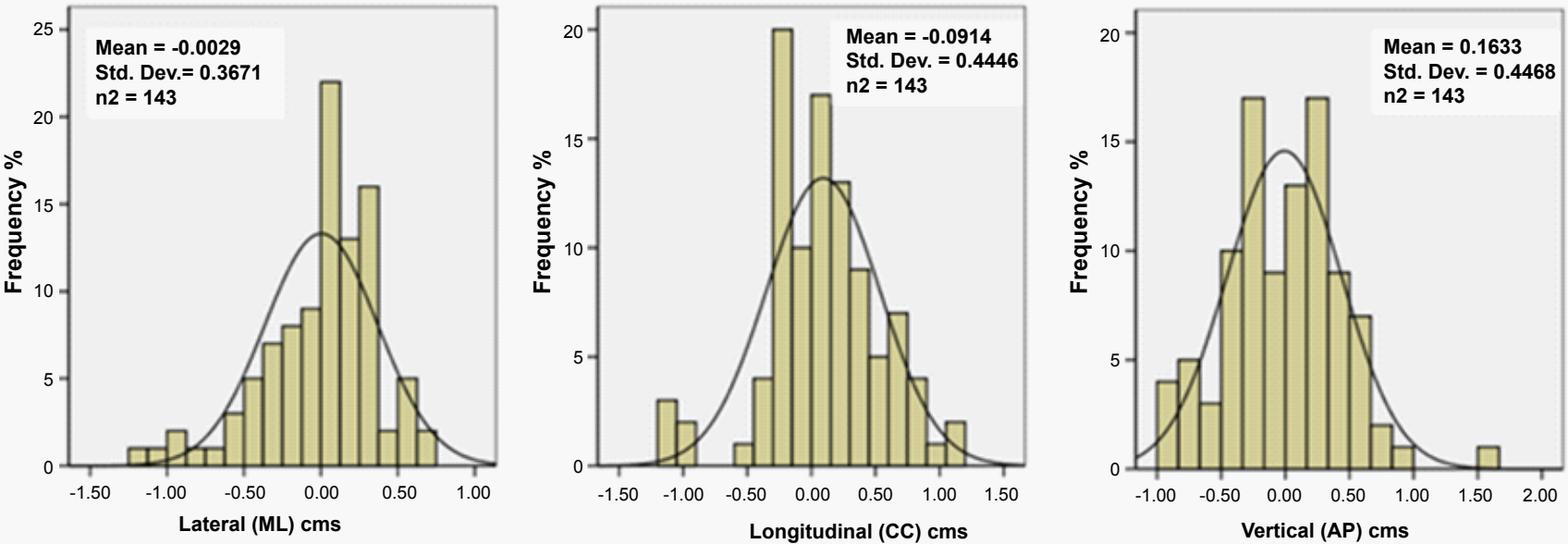

Figure 4: Measured set-up errors on Vacuum cushion in lateral (ML), longitudinal $(\mathrm{CC})$ and vertical $(\mathrm{AP})$ directions with their mean and standard deviation values.

The volumetric image registration between reference CT and real time XVI scans showed a slight higher set-up discrepancy in CC direction in both the groups, with systematic $(\Sigma)$ and random $(\sigma)$ errors $(3.8 \mathrm{~mm})$. This value is appreciably higher than errors reported in earlier studies $(\sim 3 \mathrm{~mm})$ [19-25]. But the reported studies were conducted on two dimensional digitally reconstructed radio-graphs (DRRs). The error is probably caused by the varying arm position during the daily positioning of the patient with the help of the external skin markings. While positioning a patient on the treatment couch in CC direction, the technologists align the anteriorly placed tattoo with the central 
axis of the field and also the laterally placed tattoos to the room lasers. In case of $\mathrm{BB}$, all these marks are placed on the patient, however in case of VC the lateral markings are placed on the sides of the cushion. Lateral marks are primarily used for avoiding rotational errors. The patient's arm and wrist positions may show variations daily. Due to these variations the skin at the chest level stretches slightly different every day. Thus, in the case of $\mathrm{BB}$ the errors are slightly higher than $\mathrm{VC}$. In case of VC, since the lateral markings are not placed on skin of the patient, thus the error is due to the variation of the anterior marker. The anterior marker on skin is mainly affected by the respiratory movements, which changes the $\mathrm{CC}$ alignment relative to the isocenter. The management of this discrepancy by the technologists causes daily set up variations in both the immobilization devices. In this study, we analyzed the magnitude of daily patient positioning errors for the breast patients using two different immobilization methods. Geometric errors for both the immobilization methods across all axes matches well within the applied margins used for the set up uncertainty, as demonstrated in earlier studies done at our institute [26] .

\section{Conclusion}

Both the immobilization devices, $\mathrm{BB}$ and $\mathrm{VC}$ did not show statistically significant differences in set up accuracy when used along with online guidance, thus for the delivery of intensity-modulated beams both the methods of immobilization can be considered equivalent. The mean displacement vector between the two groups was similar, which is an indication that both the groups have been equally benefited from the volumetric imaging.

Online image guidance has immense value to improve the collective mean dose delivery accuracy among a patient population, and also it is valuable in preventing large dosimetric errors for percentage of patients who have more systematic and random set up error. Because of the lack of predictive parameters to differential patient set up quality before treatment, daily XVI guidance is considered a preferable step and important safety measure in IMRT treatment. Both the systems can be used well with online guidance. Breast board is much cheaper as compared to vacloc which is associated with much more wear and tear in long term and is also higher in cost but that alone should not be the criteria to use it randomly in all patients. Patients are more comfortable in vacloc set up especially thin lean patients who would be uncomfortable in breast board's hard surfaces. We also feel very bulky and obese patients would be immobilized in a better way in vacloc although further studies should be done to verify in such patients.

\section{Acknowledgement}

We would like to acknowledge Mr M. Sree Dev of grant support and of individuals who were of direct help in the preparation of the study.

\section{References}

1. Harsolia A, Kestin L, Grills I, Wallace M, Jolly S, et al. (2007) Intensity-modulated radiotherapy results in significant decrease in clinical toxicities compared with conventional wedge-based breast radiotherapy. Int J Radiat Oncol Biol Phys 68: $1375-1380$

2. Popescu CC, Olivotto I, Patenaude V, Wai E, Beckham WA (2006) Inverseplanned, dynamic, multi-beam, intensity-modulated radiation therapy (IMRT): a promising technique when target volume is the left breast and internal mammary lymph nodes. Med Dosim 31: 283-291.

3. el-Gayed AA, Bel A, Vijlbrief R, Bartelink H, Lebesque JV (1993) Time trend of patient setup deviations during pelvic irradiation using electronic portal imaging. Radiother Oncol 26: 162-171.

4. Griffiths SE, Khoury GG, Eddy A (1991) Quality control of radiotherapy during pelvic irradiation. Radiother Oncol 20: 203-206.
5. Pignol JP, Olivotto I, Rakovitch E, Gardner S, Sixel K, et al. (2008) A multicenter randomized trial of breast intensity-modulated radiation therapy to reduce acute radiation dermatitis. J Clin Oncol 26: 2085-2092.

6. Bentel GC, Marks LB, Krishnamurthy R (1997) Impact of cradle immobilization on setup reproducibility during external beam radiation therapy for lung cancer. Int J Radiat Oncol Biol Phys 38: 527-531.

7. Creutzberg CL, Althof VG, Huizenga H, Visser AG, Levendag PC (1993) Quality assurance using portal imaging: the accuracy of patient positioning in irradiation of breast cancer. Int J Radiat Oncol Biol Phys 25: 529-539.

8. Pradier O, Schmidberger H, Weiss E, Bouscayrol H, Daban A, et al. (1999) Accuracy of alignment in breast irradiation: a retrospective analysis of clinical practice. Br J Radiol 72: 685-690.

9. van Tienhoven G, Lanson JH, Crabeels D, Heukelom S, Mijnheer BJ (1991) Accuracy in tangential breast treatment set-up: a portal imaging study. Radiother Oncol 22: 317-322.

10. de Boer HC, van Sörnsen de Koste JR, Senan S, Visser AG, Heijmen BJ (2001) Analysis and reduction of $3 \mathrm{D}$ systematic and random setup errors during the simulation and treatment of lung cancer patients with CT-based external beam radiotherapy dose planning. Int J Radiat Oncol Biol Phys 49: 857-868.

11. Erridge SC, Seppenwoolde Y, Muller SH, van Herk M, De Jaeger K, et al. (2003) Portal imaging to assess set-up errors, tumor motion and tumor shrinkage during conformal radiotherapy of non-small cell lung cancer. Radiother Oncol 66: 75-85.

12. Nelson C, Starkschall G, Chang JY (2006) The potential for dose escalation in lung cancer as a result of systematically reducing margins used to generate planning target volume. Int J Radiat Oncol Biol Phys 65: 573-586.

13. Rosenthal SA, Roach M 3rd, Goldsmith BJ, Doggett EC, Pickett B, et al. (1993) Immobilization improves the reproducibility of patient positioning during sixfield conformal radiation therapy for prostate carcinoma. Int J Radiat Oncol Biol Phys 27: 921-926

14. Soffen EM, Hanks GE, Hwang CC, Chu JC (1991) Conformal static field therapy for low volume low grade prostate cancer with rigid immobilization. Int J Radiat Oncol Biol Phys 20: 141-146.

15. Jaffray DA, Siewerdsen JH, Wong JW, Martinez AA (2002) Flat-panel conebeam computed tomography for image-guided radiation therapy. Int J Radiat Oncol Biol Phys 53: 1337-1349.

16. Islam MK, Purdie TG, Norrlinger BD, Alasti H, Moseley DJ, et al. (2006) Patient dose from kilovoltage cone beam computed tomography imaging in radiation therapy. Med Phys 33: 1573-1582.

17. Kubo HD, Hill BC (1996) Respiration gated radiotherapy treatment: a technica study. Phys Med Biol 41: 83-91.

18. Hurkmans CW, Remeijer P, Lebesque JV, Mijnheer BJ (2001) Set-up verification using portal imaging; review of current clinical practice. Radiother Oncol 58: 105-120.

19. Deutsch M, Bryant J, Bass G (1999) Radiotherapy review on national surgical adjuvant breast and bowel project (NSABP) phase III breast cancer trials: is there a need for submission of portal/simulation films. Am J Clin Oncol 22 606-608.

20. Halperin R, Roa W, Field M, Hanson J, Murray B (1999) Setup reproducibility in radiation therapy for lung cancer: a comparison between $\mathrm{T}$-bar and expanded foam immobilization devices. Int J Radiat Oncol Biol Phys 43: 211-216.

21. Holmberg O, Huizenga H, Idzes MH, Lebesque JV, Vijlbrief RE, et al. (1994) In vivo determination of the accuracy of field matching in breast cancer irradiation using an electronic portal imaging device. Radiother Oncol 33: 157-166.

22. Idzes MH, Holmberg O, Mijnheer BJ, Huizenga H (1998) Effect of set-up uncertainties on the dose distribution in the match region of supraclavicular and tangential breast fields. Radiother Oncol 46: 91-98.

23. Kelly CA, Wang XY, Chu JC, Hartsell WF (1996) Dose to contralateral breast: a comparison of four primary breast irradiation techniques. Int J Radiat Oncol Biol Phys 34: 727-732.

24. Lirette A, Pouliot J, Aubin M, Larochelle M (1995) The role of electronic porta imaging in tangential breast irradiation: a prospective study. Radiother Oncol 37: 241-245. 
Citation: Jassal K, Bisht S, Kataria T, Sachdev K, Choughle A, et al. (2013) Comparison of Geometrical Uncertainties in Breast Radiation Therapy with Different Immobilization Methods. J Nucl Med Radiat Ther 4: 140. doi:10.4172/2155-9619.1000140

Page 6 of 6

25. Pouliot J, Lirette A (1996) Verification and correction of setup deviations in tangential breast irradiation using EPID: gain versus workload. Med Phys 23 1393-1398.
26. Kataria T, Abhishek A, Chadha P, Nandigam J (2011) Set-up uncertainties: online correction with X-ray volume imaging. J Cancer Res Ther 7: 40-46. 